\title{
Neuronal firing patterns outweigh circuitry oscillations in parkinsonian motor control
}

\author{
Ming-Kai Pan, ${ }^{1,2,3}$ Sheng-Han Kuo, ${ }^{4}$ Chun-Hwei Tai, ${ }^{2}$ Jyun-You Liou, ${ }^{5}$ Ju-Chun Pei, ${ }^{6}$ Chia-Yuan Chang, ${ }^{6}$ Yi-Mei Wang, ${ }^{3}$ \\ Wen-Chuan Liu, ${ }^{2}$ Tien-Rei Wang, ${ }^{2}$ Wen-Sung Lai, ${ }^{6,7}$ and Chung-Chin Kuo ${ }^{2,7,8}$ \\ 'Department of Medical Research and ${ }^{2}$ Department of Neurology, National Taiwan University Hospital, Taipei, Taiwan. ${ }^{3}$ Department of Neurology, National Taiwan University Hospital, Yun-Lin Branch, \\ Yun-Lin, Taiwan. ${ }^{4}$ Department of Neurology, College of Physicians and Surgeons, and ${ }^{5}$ Department of Physiology and Cellular Biophysics, Columbia University, New York, New York, USA. ${ }^{6}$ Department of \\ Psychology, National Taiwan University, Taipei, Taiwan. ${ }^{7}$ Neurobiology and Cognitive Science Center and ${ }^{8}$ Department of Physiology, National Taiwan University, Taipei, Taiwan.
}

\begin{abstract}
Neuronal oscillations at beta frequencies $(20-50 \mathrm{~Hz})$ in the cortico-basal ganglia circuits have long been the leading theory for bradykinesia, the slow movements that are cardinal symptoms in Parkinson's disease (PD). The beta oscillation theory helped to drive a frequency-based design in the development of deep brain stimulation therapy for PD. However, in contrast to this theory, here we have found that bradykinesia can be completely dissociated from beta oscillations in rodent models. Instead, we observed that bradykinesia is causatively regulated by the burst-firing pattern of the subthalamic nucleus (STN) in a feedforward, or efferent-only, mechanism. Furthermore, STN burst-firing and beta oscillations are two independent mechanisms that are regulated by different NMDA receptors in STN. Our results shift the understanding of bradykinesia pathophysiology from an interactive oscillatory theory toward a feed-forward mechanism that is coded by firing patterns. This distinct mechanism may improve understanding of the fundamental concepts of motor control and enable more selective targeting of bradykinesia-specific mechanisms to improve PD therapy.
\end{abstract}

\section{Introduction}

A cardinal feature of Parkinson's disease (PD) is slow movements, known as bradykinesia. The neuronal activities related to bradykinesia are two electrophysiological landmarks in PD: oscillations, the pathological augmentation of cerebral field activities in beta frequencies $(20-50 \mathrm{~Hz})$ between cortex and subthalamic nucleus (STN) (1-9), versus codes, the excessive burst-firing patterns in STN (10-13). The leading hypothesis has long been that beta oscillations underlie bradykinesia, supported by the fact that beta power correlates with bradykinesia severity (6-9) and injecting beta electric activities into cortex $(14,15)$ and STN $(13,16)$ worsens motor performances. The oscillatory theory has deeply impacted PD therapy development and has served as important conceptual basis for deep brain stimulation (DBS) (16-18).

However, we recently found that excessive STN bursts, the abnormal codes in PD, can also lead to bradykinesia $(10,11)$. The generation of STN bursts requires T-type calcium channels (CaTs), which are the intrinsic ion channels in STN serving as burst initiator (19). The cortex regulates STN bursts via NMDAergic cortico-subthalamic transmission (12), which also generates beta oscillations (12). The new understandings of the online modulatory mechanism of both STN bursts and beta oscillations open the window to approaching the fundamental question, what is the mechanism directly responsible for bradykinesia: the frequencydependent oscillations or STN bursting codes? We therefore applied online modulations by selectively manipulating STN

Conflict of interest: The authors have declared that no conflict of interest exists. Submitted: April 20, 2016; Accepted: September 22, 2016.

Reference information: / Clin Invest. 2016;126(12):4516-4526. doi:10.1172/JCI88170. bursts and beta oscillations in 6-hydroxydopamine (6-OHDA) hemiparkinsonian rat models $(10-13,20)$ and investigated their effects on bradykinesia (Figure 1, Supplemental Figure 1, and Supplemental Video 1; supplemental material available online with this article; doi:10.1172/JCI88170DS1).

\section{Results}

Modulating intrinsic burst-firing properties of STN dissociates beta oscillations from bradykinesia. Beta oscillations and STN bursts, the two bradykinesia-generating candidates, either can work synchronously in a cascade, or one of them is an epiphenomenon. We first manipulated STN bursts while observing beta oscillations and bradykinesia. Taking advantage of our previous studies $(10,11)$, we suppressed or facilitated STN bursts by manipulating CaTs. Applying CaT blockers $\left(\mathrm{NiCl}_{2}\right.$ and mibefradil) into STN suppressed STN bursts and also remedied bradykinesia in 6-OHDA hemiparkinsonian rats (Figure 2, A-F, Supplemental Figure 2, and Supplemental Video 2). However, the oscillatory profiles remained unchanged, including in situ beta synchronization of STN and cortex (Figure 2, G and H) and cortico-subthalamic oscillations (Figure 2, I and J) in both resting and moving conditions. These results clearly demonstrated that STN bursts do not cause bradykinesia via beta oscillations, and oscillation and bradykinesia could be dissociated in PD models. Consistently, application of constant hyperpolarizing current (HC) into STN can increase CaT availability (10) and burst discharges (Figure 3, A and B), which sufficiently recapitulated bradykinesia in normal rats without generating beta oscillations (Figure 3, C-J). Instead, HC further suppressed regional beta power in STN (Figure $3 \mathrm{G}$ ), suggesting that $\mathrm{HC}$ augments automaticity of individual 
A

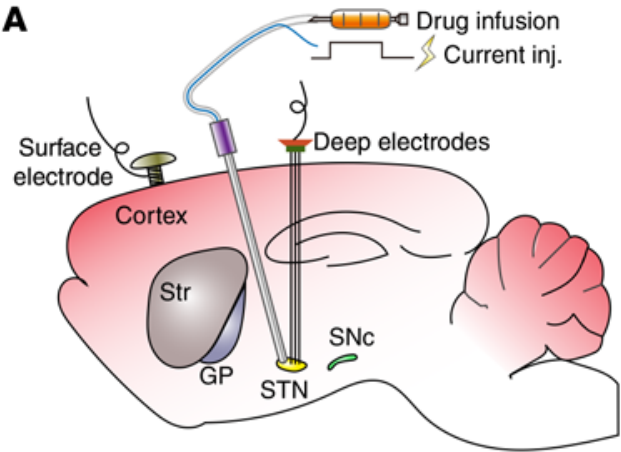

Locomotor behaviors Single-unit recordings (STN) Local field potentials

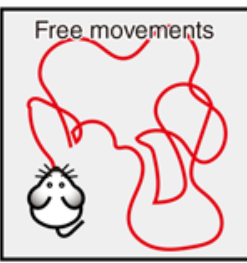

Forced movements (Rotarod)
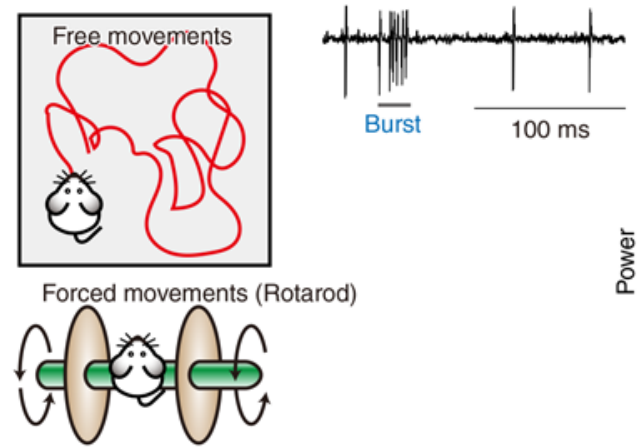

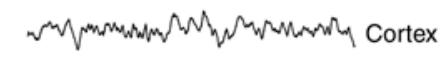

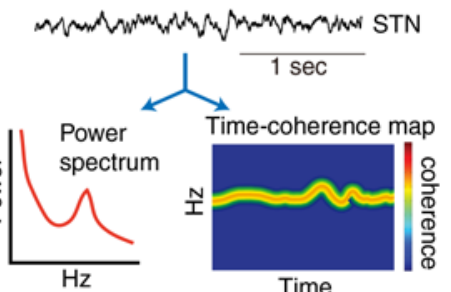

C
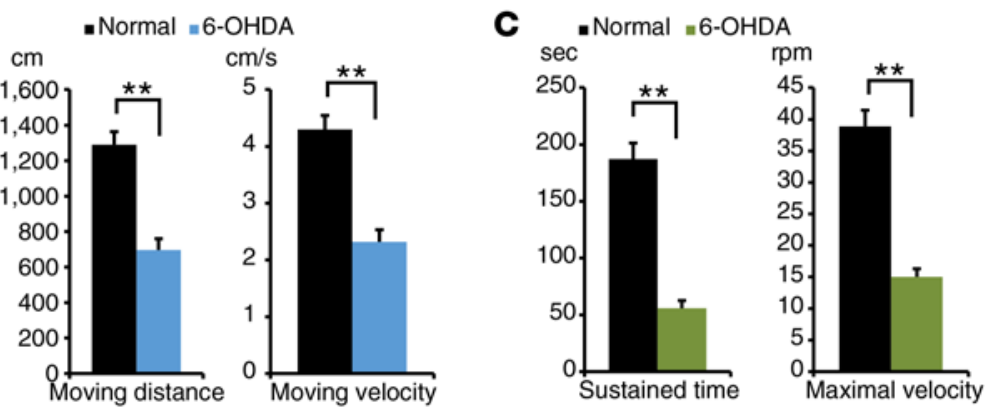

D

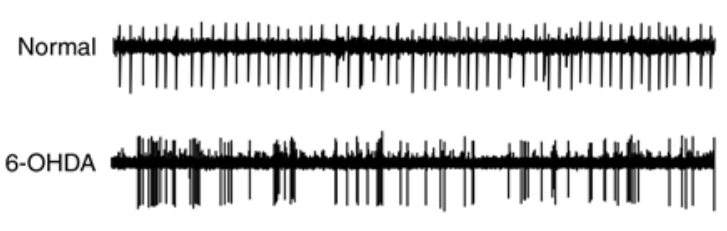

$500 \mathrm{~ms}$
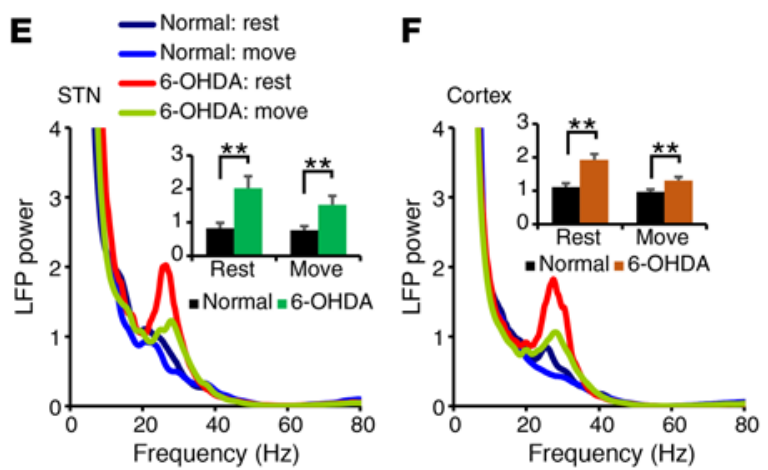
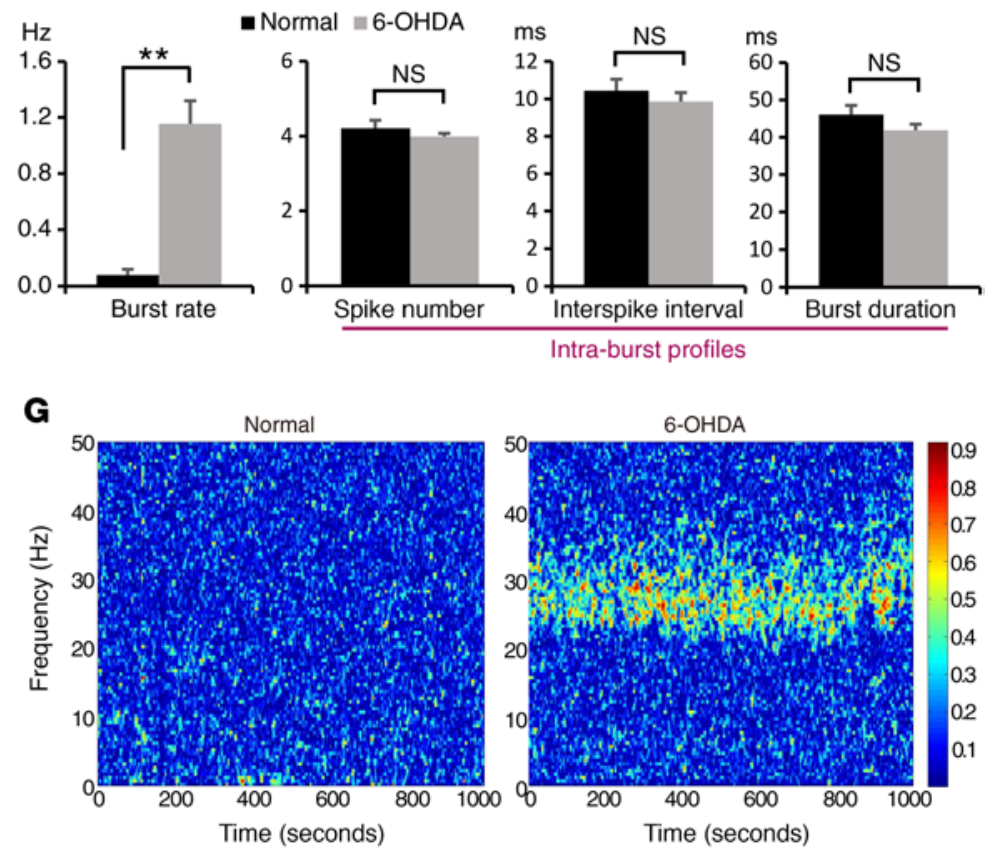

Figure 1. Behavioral and real-time neuronal abnormalities in 6-OHDA-lesioned parkinsonian rat model. (A) Scheme of the experimental design. Rats received surgical placements of drug infusion cannula coupled with a stimulating electrode, and recording electrodes in STN and cortex. Implanted rats received part or all of the following evaluations, including locomotor behaviors (open-field free movements and rotarod forced movements), single-unit recordings, and LFPs, before and after pharmacological and/or electrical manipulations. inj., injection; Str, striatum; GP, globus pallidus; SNc, substantia nigra pars compacta. (B and C) Locomotor behaviors. 6-OHDA rats developed motor deficits, especially slow movements (bradykinesia), in both (B) free-moving and (C) forced-moving paradigms ( $n=11$ in both paradigms). (D) STN firing patterns. 6-OHDA rats developed excessive burst firings in STN, while the intra-burst profiles remained unchanged $(n=10)$. ( $\mathbf{E}-\mathbf{C})$ Oscillatory profiles. ( $\mathbf{E}$ and $\mathbf{F})$ In situ synchronization of oscillatory activities presented as LFPs. STN and cortical power in beta frequencies $(20-50 \mathrm{~Hz})$ were pathologically increased in both resting and moving conditions in $6-0 \mathrm{HDA}$ rats $(n=11)$. (C) Long-range cortico-subthalamic oscillations presented by time-coherence plot. 6-OHDA rats developed robust oscillations in beta frequencies ( $n=11$ ). Statistical analyses were performed using a nonparametric Wilcoxon signed-rank test. Data are presented as mean \pm SEM; ${ }^{* *} P<0.01$. 

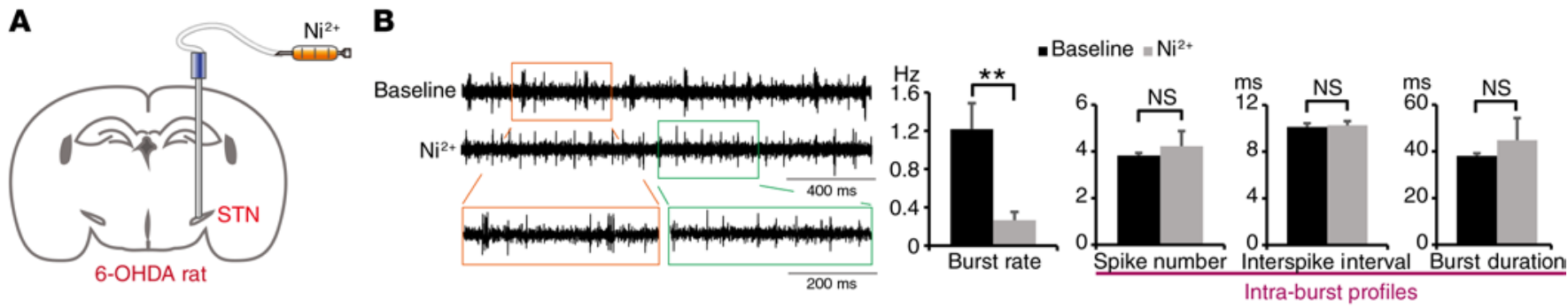

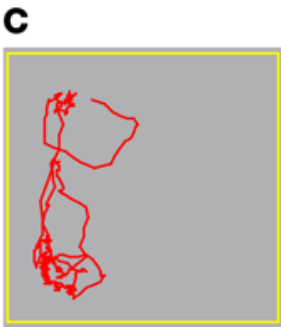

6-OHDA: Baseline

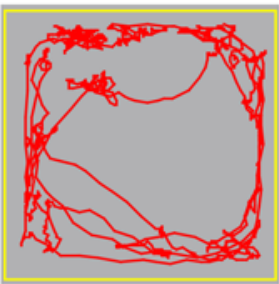

6-OHDA: $\mathrm{Ni}^{2+}$
D

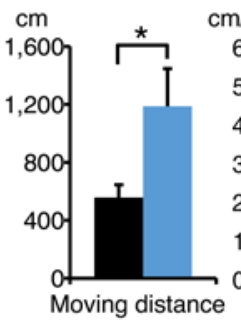

Baseline $\mathrm{Ni}^{2+}$

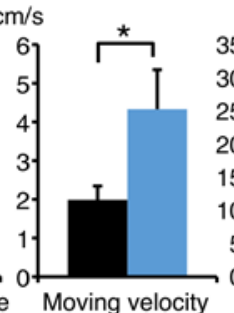

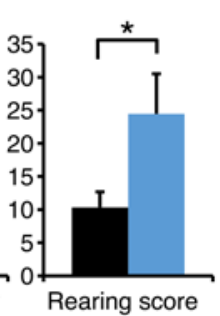

$\mathbf{E}$
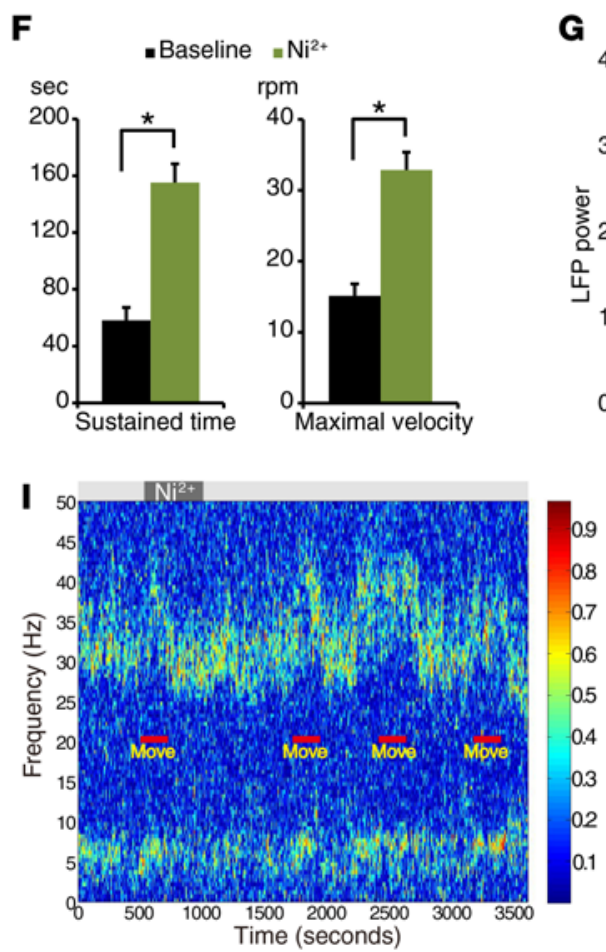

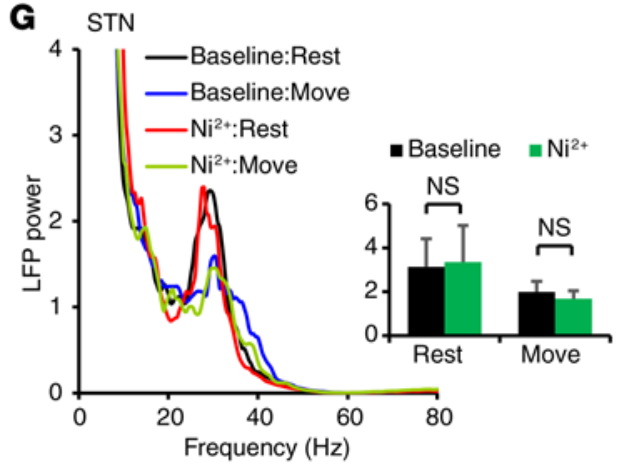

H Cortex

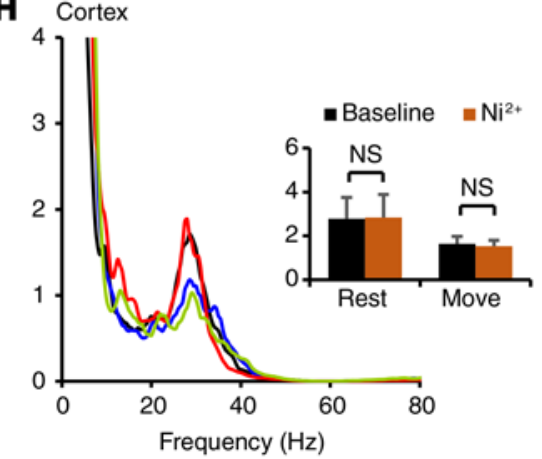

Figure 2. Suppression of burst-generating capacity in STN rescues bradykinesia but not beta oscillations in 6-OHDA-lesioned hemiparkinsonian rats. (A) Subthalamic infusion of $\mathrm{NiCl}_{2}\left(\mathrm{Ni}^{2+}\right)$, a T-type calcium channel blocker, in 6-OHDA rats. (B) Sample sweeps of single-unit recordings and quantitative burst analysis. $\mathrm{Ni}^{2+}$ suppressed burst firings without changing the intra-burst profiles in 6-OHDA rats $(n=25)$. (C-F) Behavioral assessments. (C) Typical traces showing $\mathrm{Ni}^{2+}$ effects in free-moving activities. $\mathrm{Ni}^{2+}$ ameliorated (D) motion difficulties and $(\mathbf{E})$ asymmetries $(n=9)$. Note that moving velocity was rescued in both $(\mathbf{D})$ free-moving and $(\mathbf{F})$ forced-moving $(n=8)$ paradigms. (G-J) Oscillatory profiles. $(\mathbf{G}$ and $\mathbf{H})$ In situ synchronization. Ni ${ }^{2+}$ had no effect on STN or cortical powers in beta frequency $(20-50 \mathrm{~Hz})$ in both rest and moving conditions. Quantitative analysis in these rats showed no change in beta power (bar plots, $n=11$ ). (I) Long-range cortico-subthalamic oscillations. Dark gray section of the bar above indicates Ni² infusion. (J) Quantitative analysis of coherence shows that $\mathrm{Ni}^{2+}$ did not change the pathological state of interlocking power (right panel) or frequency (left panel) in beta ranges. Statistical analyses were performed using a nonparametric Wilcoxon signed-rank test. Data are presented as mean $\pm \mathrm{SEM} ;{ }^{*} P<0.05,{ }^{* *} P<0.01$.

STN neurons, and therefore weakens synchronization between nearby neurons and suppresses beta power. These results provided direct evidence that STN bursts, not beta oscillations, are the immediate mechanism of bradykinesia. STN bursts are either the downstream player in the bradykinesia-generating cascade or an isolated mechanism independent of beta oscillations.
Bursting codes and beta oscillations are mediated by different NMDA receptor subtypes in STN. We then examined whether beta oscillations are the upstream regulator of STN bursts in the bradykinesia-generating cascade. Beta oscillations depend on NMDAergic cortico-subthalamic transmission (12); we thus selectively inhibited NMDA receptor (NMDAR) containing 
A

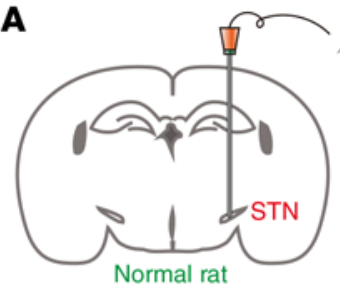

C

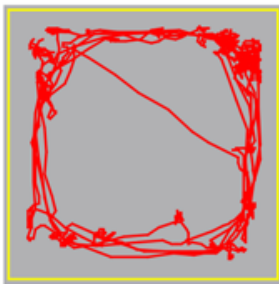

Normal-Baseline
Constant $\mathrm{HC}$

Baseline

$\mathrm{HC}$

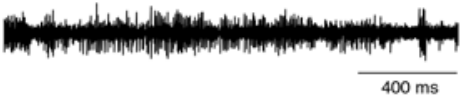

B

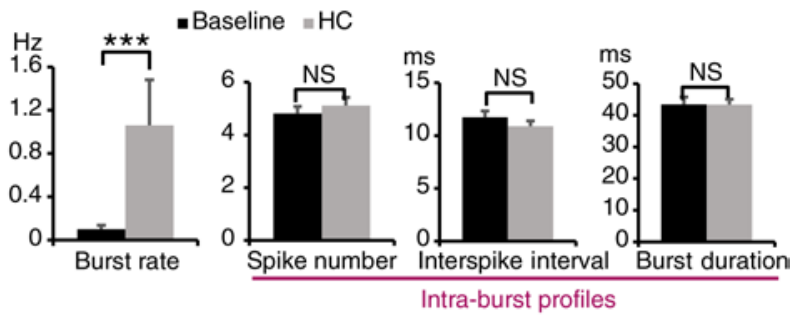

D

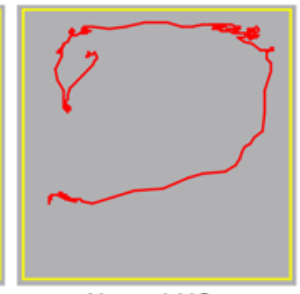

Normal-HC
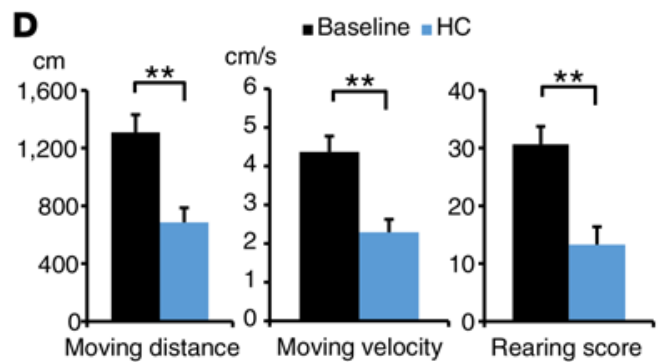

E

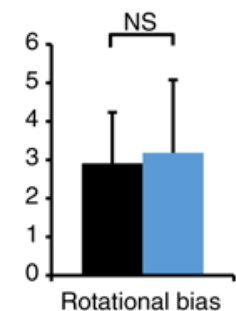

$\mathbf{F}$

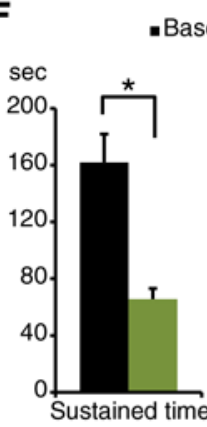

- Baseline $=\mathrm{HC}$
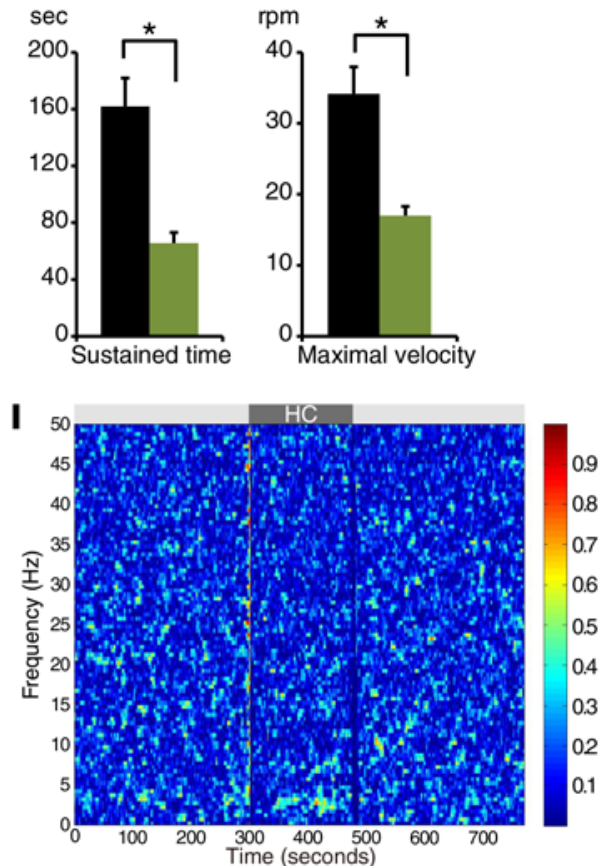

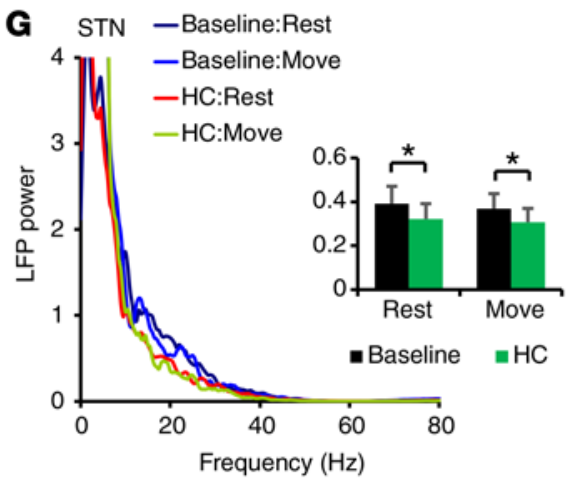

H Cortex

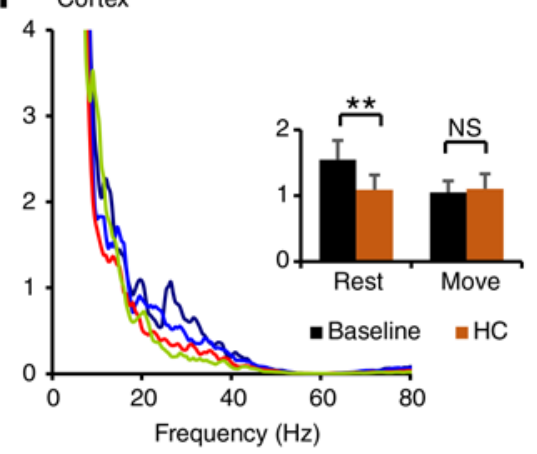

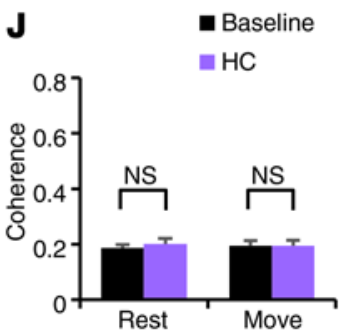

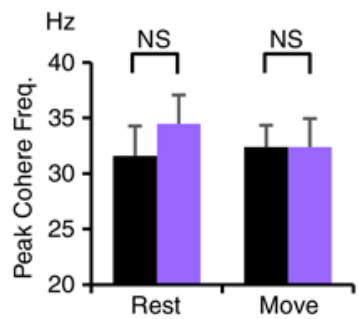

Figure 3. Augmentation of burst-generating propensity in STN recapitulates bradykinesia but not beta oscillations in normal rats. (A) Scheme showing subthalamic application of constant HCs in normal rats. (B) HC increased burst rates in STN $(n=15)$ and left intra-burst profiles unchanged. (C-F) Behavioral assessments. (C) Typical free-moving traces showing HC effects. HC transformed normal rats into hemiparkinsonian states and recapitulated (D) motion difficulties and $(\mathbf{E})$ asymmetries $(n=11)$. The capacity of fast movements was also compromised in $(\mathbf{F})$ the forced-moving paradigm $(n=6)$. $(\mathbf{G}-\mathbf{J})$ Oscillatory profiles. (G) HC further suppressed LFPs in STN, instead of reinforcing beta power mimicking the parkinsonian state $(n=13)$. (H) HC also remotely suppressed cortical beta power. (I and J) The cortico-subthalamic oscillations remained unsynchronized. Statistical analyses were performed using a nonparametric Wilcoxon signed-rank test. Data are presented as mean $\pm \mathrm{SEM} ;{ }^{*} P<0.05,{ }^{* *} P<0.01,{ }^{* * *} P<0.001$.

the GluN2A subunit, which has the fastest kinetics in the beta range (21). Subthalamic application of CPP, a GluN2A antagonist, markedly suppressed oscillatory profiles in 6-OHDA rats but had no effect on either bradykinesia or STN bursts (Figure 4, A-D, and Supplemental Figure 3, A-D). The results directly showed that beta oscillations are not involved in the genesis of STN bursts. Therefore, beta oscillations are not the upstream regulator in the bradykinesia-generating cascade.
We then examined the roles of the GluN2B and GluN2D subunits. Blockers (Ro 25-6891 [RO] and PPDA) of the GluN2B/D receptors specifically suppressed STN bursts and ameliorated bradykinesia, but oscillatory profiles remained unchanged (Figure 4, Supplemental Figures 3 and 4, and Supplemental Video 3). These results demonstrated that bursting codes and beta oscillations are two parallel mechanisms regulated by different NMDARs in STN. In striking contrast to the oscillatory 


\section{GluN2A inhibition}

A

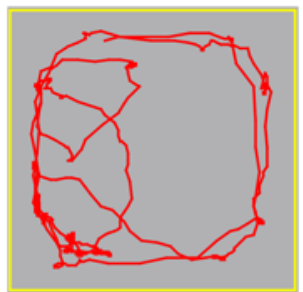

Baseline

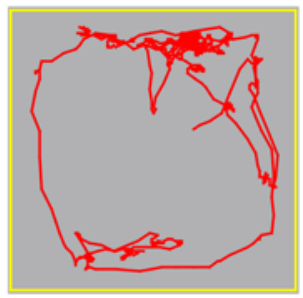

CPP

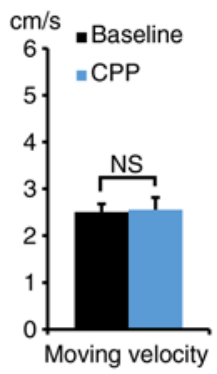

B

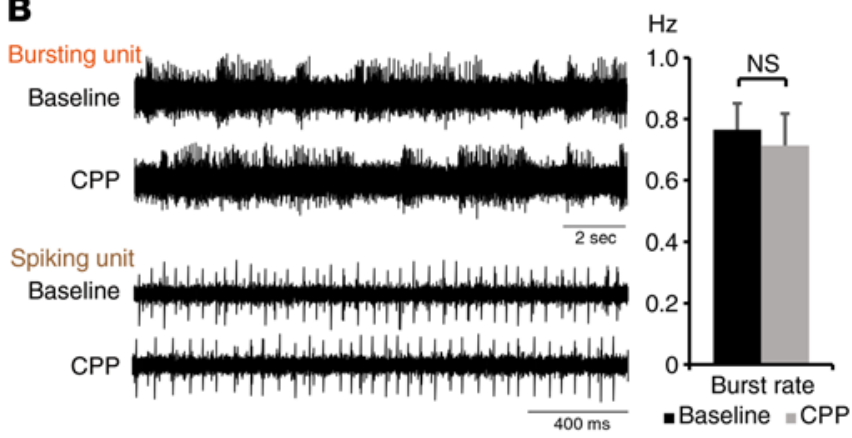

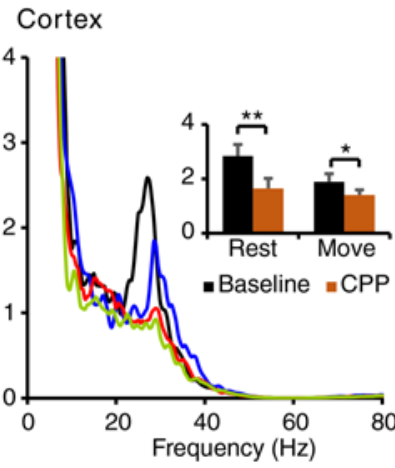
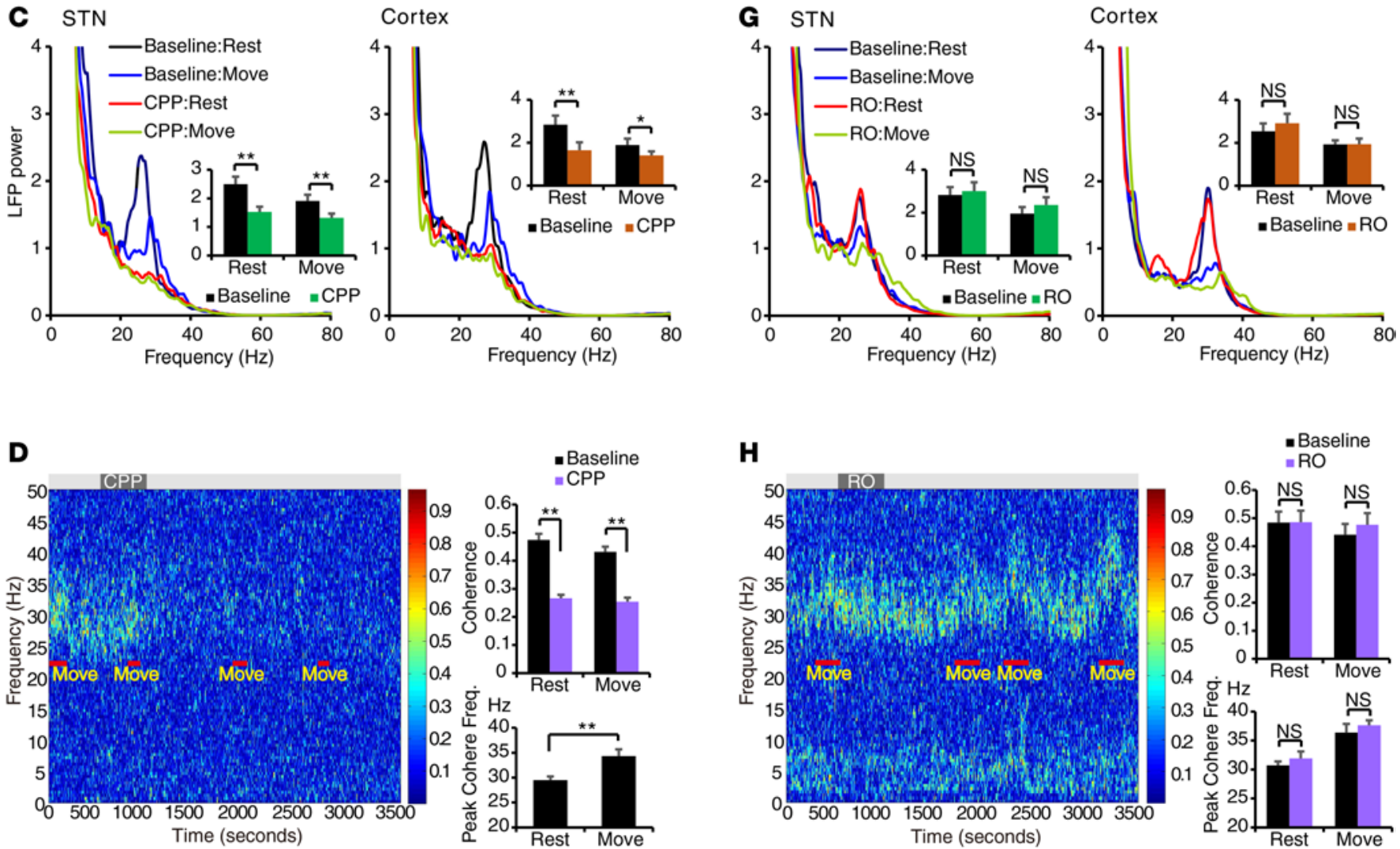

Figure 4. Differential contributions of NMDAR subtypes in bradykinesia and electrophysiological profiles of 6-OHDA rats. (A-D) CPP, a selective GluN2A blocker, had no effect on (A) locomotor behaviors $(n=18)$ or (B) STN burst firings $(n=29)$, but dramatically reduced both (C) in situ synchronization and (D) cortico-subthalamic oscillations $(n=9)$. (E-H) RO, a selective GluN2B/D blocker, preferentially inhibiting GluN2B subunit, rescued (E) parkinsonian motor deficits $(n=13)$, suppressed (F) pathological bursts in STN $(n=38)$, but had no effect on $(\mathbf{G})$ in situ synchronization or (H) cortico-subthalamic oscillations $(n=11)$. Also refer to Supplemental Figure 3 for additional behavioral and single-unit profiles. Statistical analyses were performed using a nonparametric Wilcoxon signed-rank test. Data are presented as mean $\pm \mathrm{SEM} ;{ }^{*} P<0.05,{ }^{* *} P<0.01$. 
A

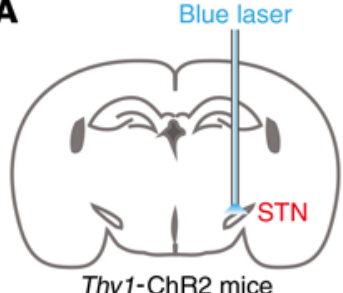

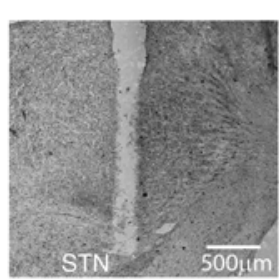

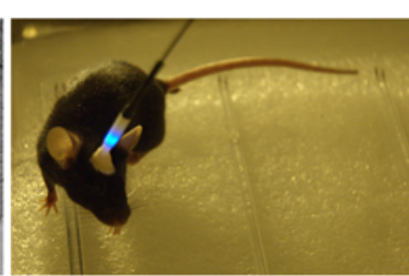

B
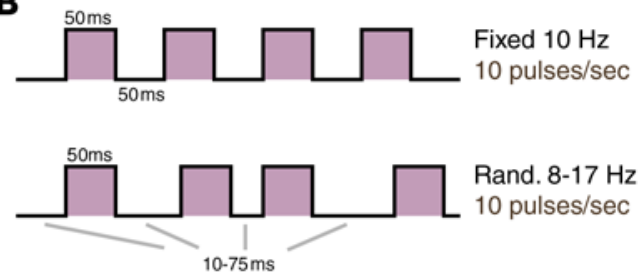

C

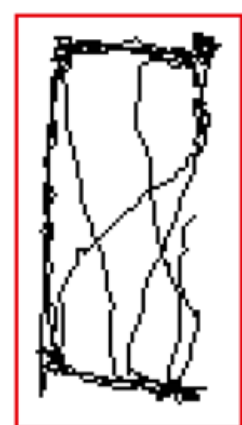

Light off

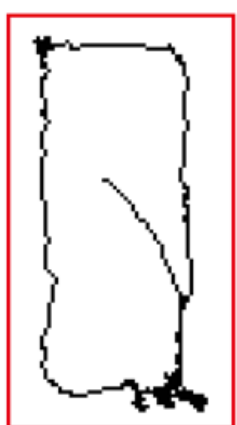

Light Rand

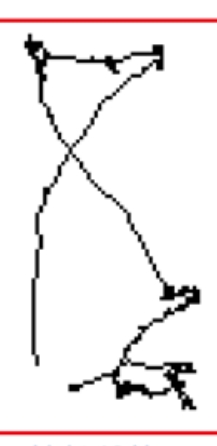

Light-10 Hz
$\mathbf{F}$

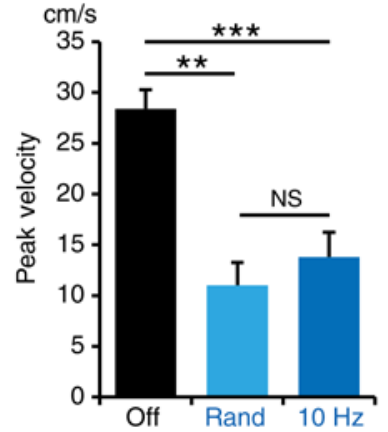

G

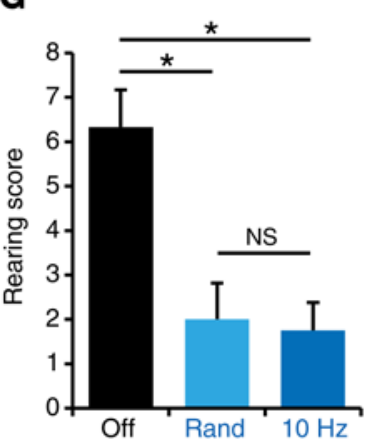

D

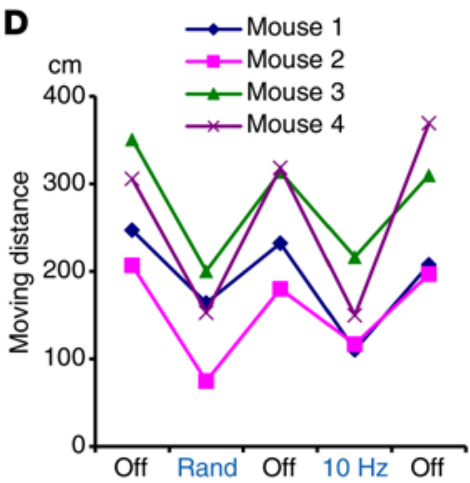

E

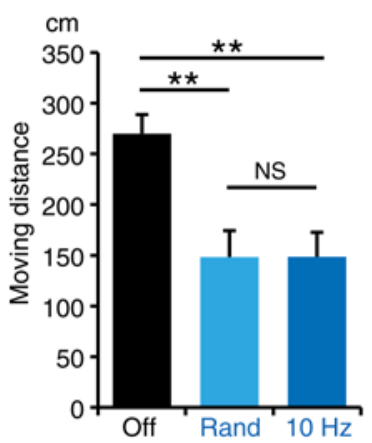

H

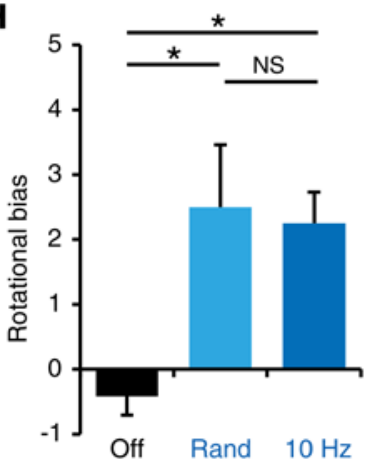

I

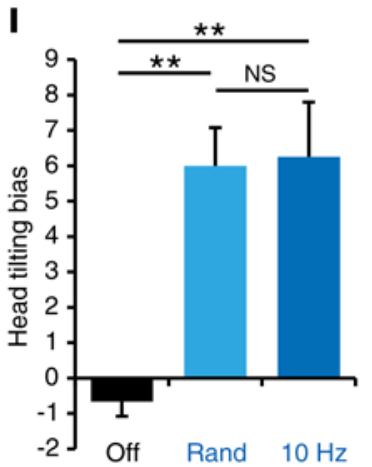

Figure 5. Bradykinesia is independent of the regularity of cortico-subthalamic transmissions. (A) Schematic illustration of fiber optic cannula implanted in STN for the stimulation of cortico-subthalamic axonal terminals in Thy1-ChR2 mice. (B) Illustration of two illumination protocols: fixed 10 Hz stimulation and randomized frequency shuffles with the same stimulation loads (10 pulses/s). (C-I) Sample traces of locomotor behaviors and corresponding statistic results, showing that fixed-frequency and randomized (Rand) stimulation recapitulated bradykinesia with similar severity, quantified by (E-G) motion difficulties and ( $\mathbf{H}$ and $\mathbf{I})$ asymmetries $(n=4)$. Also refer to Supplemental Figure 5 for thermodynamic controls, which followed the same protocols with non-activating yellow light $(589 \mathrm{~nm})$ laser. Statistical analyses were performed using 1-way ANOVA with post-hoc Bonferroni correction. Data are presented as mean $\pm \mathrm{SEM}$; ${ }^{*} P<0.05,{ }^{* *} P<0.01,{ }^{* *} P<0.001$.

hypothesis, beta oscillations are not even involved in the bradykinesia-generating cascade and STN bursts play an independent role in bradykinesia.

Bradykinesia is regulated by STN bursts via a feed-forward mechanism. We next investigated the mechanisms by which STN bursts could lead to bradykinesia. Beyond beta oscillations, STN bursts may still involve other forms of frequency-dependent mechanisms. Without the phase synchronicity of nearby neurons detected as beta oscillations, individual STN neurons may still require regular NMDAergic inputs to generate meaningful bursts and thus bradykinesia. To evaluate whether transsynaptic regularity modulates bradykinesia, we optogenetically activated cortico-subthalamic axonal terminals by illuminating STN in Thy1-ChR2 transgenic mice $(12,13)$ (Figure 5A) with either fixed-frequency $(10 \mathrm{~Hz})$ stimulation or frequency-independent shuffles (Figure 5B). The two stimulation protocols were of the same stimulation loads (10 pulses/s) and generated robust and similar motor deficits in normal mice (Figure 5, C-I, Supplemental Figure 5, and Supplemental Video 4). The results indicated that motor inhibition is not only dissociated from low-frequency oscillations of grouped neuronal activities, but also independent of the regularity of action potentials transmitted in the corticosubthalamic axons.

The timing of shuffled illumination was completely artificial and unpredictable, and therefore minimized the opportunity for the circuitry to adapt from the feedback interaction. Our results strongly suggested that once STN bursts are generated, the circuitry passes this information via a feed-forward mechanism to the downstream nuclei and no longer requires continuous NMDAergic monitoring from cortex to STN. To test this hypothesis, we gave HC to generate STN bursts while simultaneously blocking NMDAergic cortico-subthalamic transmission (Figure 
A

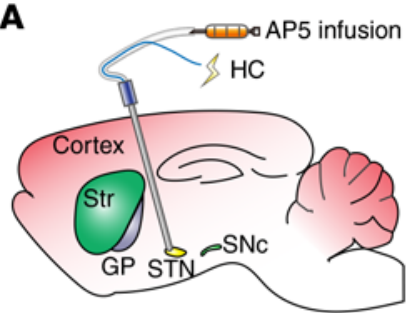

Normal rat
B

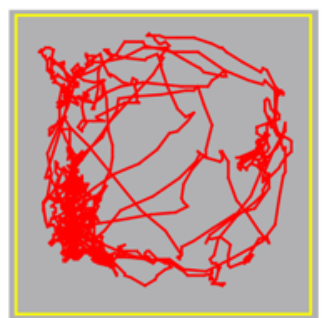

Baseline

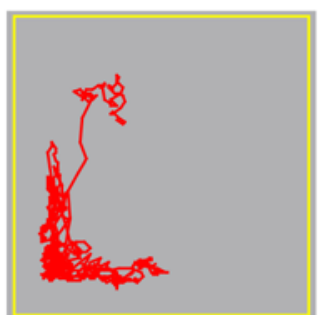

$\mathrm{HC}$

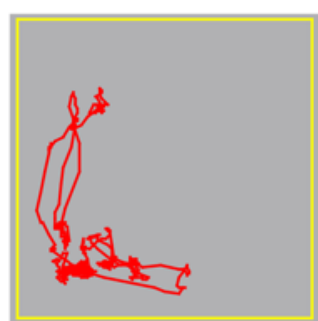

HC \& AP5
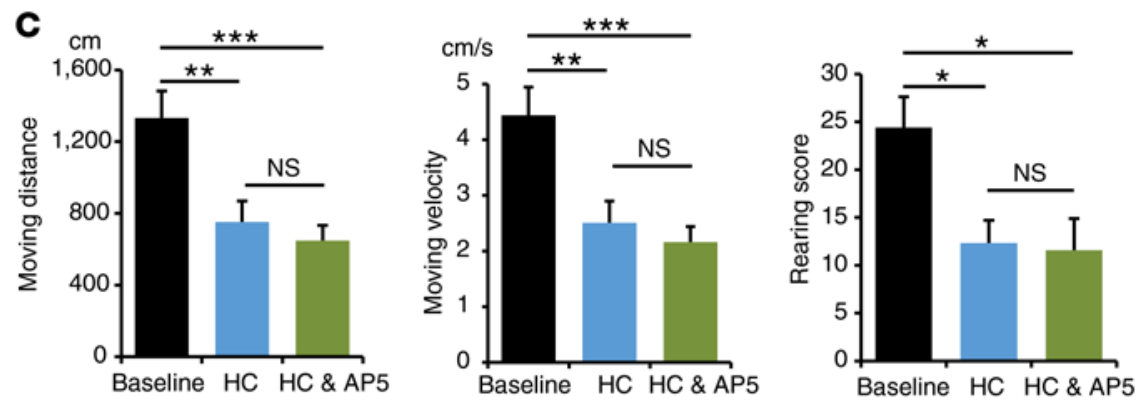

D

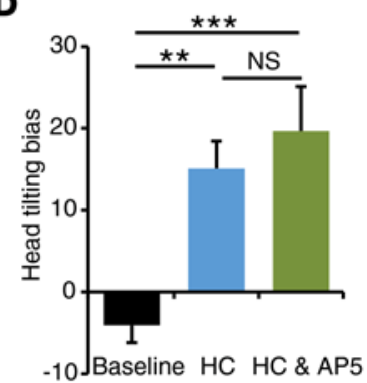

E

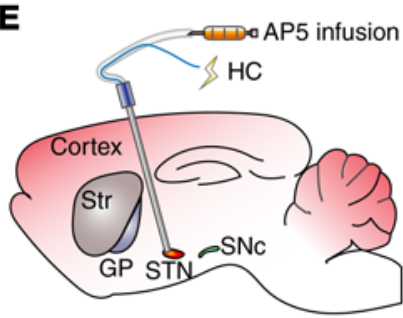

6-OHDA rat

$\mathbf{F}$

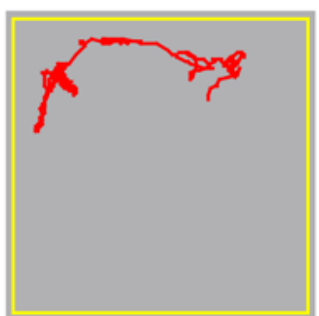

Baseline

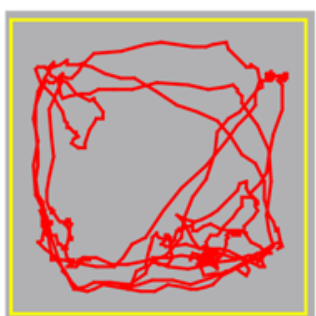

AP5

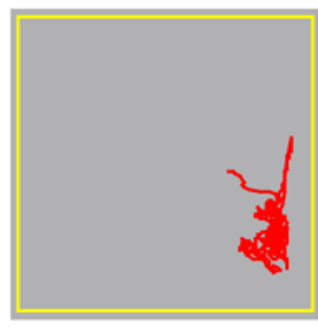

AP5 \& HC
G

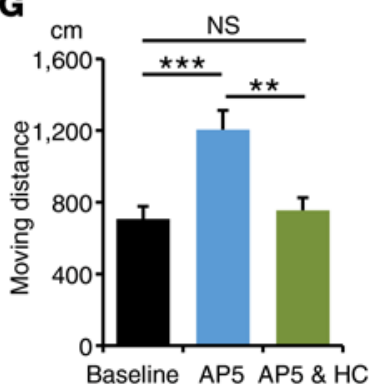

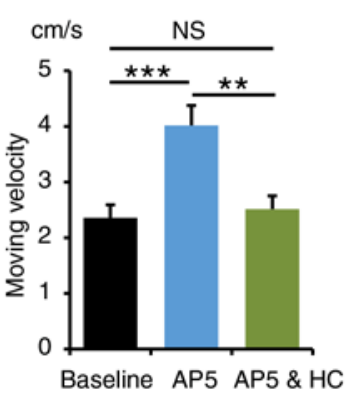

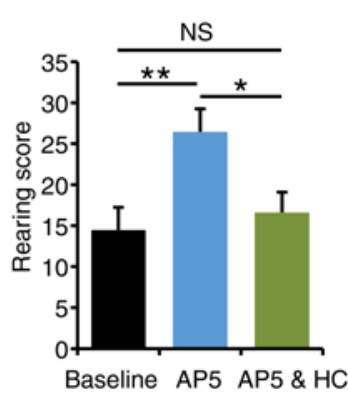

H

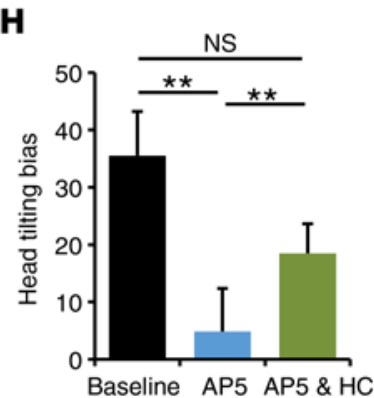

Figure 6. Bradykinesia is independent of the continuity of NMDAergic cortico-subthalamic transmissions. (A) Schematic illustration of HC application in STN of a normal rat, with or without simultaneous microinfusion of AP5, a nonselective NMDAR blocker. (B-D) Sample traces and quantitative analysis of locomotor activities. Bradykinesia can be recapitulated in normal rats by subthalamic HC application, but additional NMDAergic cortico-subthalamic interruption cannot reverse bradykinesia $(n=12)$. (E-H) Similar settings in 6-OHDA rats, showing that the therapeutic effect of NMDAergic interruption by AP5 can be abolished by additional HC application in STN $(n=15)$. Statistical analyses were performed using 1-way ANOVA with post-hoc Bonferroni correction. Data are presented as mean $\pm \mathrm{SEM} ;{ }^{*} P<0.05,{ }^{* *} P<0.01,{ }^{* *} P<0.001$.

6A). HC sufficiently induced STN bursts and bradykinesia in normal animals, but the bradykinesia-generating effect of $\mathrm{HC}$ could not be rescued by interrupting NMDAergic cortico-subthalamic transmission (Figure 6, B-D, and Supplemental Figure 6A). The same principles also apply to 6-OHDA rats, which had excessive STN bursts (Figure 1D and Supplemental Figure 7A) (10-12). Blocking NMDAergic transmission in STN suppressed bursts and rescued bradykinesia. However, HC sufficiently restored STN bursts and eliminated the therapeutic effects of
NMDAR blockers (Figure 6, E-H, and Supplemental Figure 7). Once more, animal behaviors in both normal and 6-OHDA rats only aligned with STN firing patterns. Oscillatory profiles either remained unchanged or were contrary to the profiles that would be predicted by the current oscillatory theory (Supplemental Figures 6 and 7). These results clearly indicated that once STN bursts are generated, bradykinesia no longer requires ongoing cortical regulation, regardless of its synchronicity (Figures 2-4), regularity (Figure 5), or continuity (Figure 6). These characteris- 
tics reveal a delicate feed-forward role of STN bursts in bradykinesia, and are compatible with the fast-acting and quick-responsive nature of motor execution. They are also consistent with the race model of basal ganglia circuity in the STN-substantia nigra pars reticulata (SNr) axis (22), which is immediately downstream from the STN. The interruption of planned actions in the STN/SNr axis has a critical gate of timing, and intervention beyond this time point fails to stop motor execution (22). In contrast to the feed-forward mechanism shown in bradykinesia, beta oscillations have a feedback nature that requires continuous reciprocal interactions in the circuitry. Inhibition of GluN2A transmission at the cortico-subthalamic terminals in STN sufficiently disrupted beta power in the "upstream" cortex (Figure 4C). Consistently, HC suppressed beta power in STN locally and also remotely in the cortex (Figure $3, \mathrm{G}$ and $\mathrm{H}$ ).

Taken together, our results show that STN bursts control bradykinesia via a feed-forward mechanism. Nevertheless, it should be noted that we focused on the fastest cortico-subthalamic "hyperdirect" pathway in this study. It is evident that the slower indirect pathway eventually gets involved and tunes the motor behaviors in the later steps (20). It should also be noted that this study targeted bradykinesia, a cardinal involuntary movement in PD. Although not related to bradykinesia, beta oscillations seemed to reflect the volitional aspect of motor decision states (moving versus resting states; Figures 2 and 4), regardless of the motor performances with or without NMDAR/CaT modulations (see Discussion).

\section{Discussion}

We discovered that bradykinesia is regulated by STN bursting codes in a feed-forward mechanism and can be completely dissociated from beta oscillations. STN bursts and beta oscillations are two parallel mechanisms controlled by different NMDARs in STN. In this study, we quantified bradykinesia (slow movements) by velocity measurement, which has been reliably used in other studies in the 6-OHDA model (20,23-26). Nevertheless, there are other behavioral tests linked to bradykinesia and other motor deficits in PD $(27,28)$, and these deserve further investigation.

Differential distributions of NMDARs and CaTs in STN and their potential impacts. The burst-generating cascade requires the collaboration between GluN2B/D NMDARs and CaTs in STN, while beta oscillations depend on GluN2A NMDARs. Consistent with the clear-cut dissociation in electrophysiology, we found differential distributions of NMDAR subtypes in the STN neurons of 6-OHDA rats (Supplemental Figure 8). The oscillation-contributing GluN2A subunits were diffusely expressed in STN soma (Supplemental Figure 8, A-C), while the burst-generating GluN2B/D subunits had punctal expression extended to STN neurites (Supplemental Figure 8, D-I). Also, both GluN2B/D and CaTs (e.g., $\mathrm{Ca}_{\mathrm{v}} 3.3$, the predominant CaT subtype in STN) (29) had the characteristic punctal pattern and distribution (Supplemental Figure 9), supporting their collaborative role in burst generation. It is interesting that NMDAR subtypes and CaTs are differentially segregated according to their burst- or oscillation-generating roles. Beta oscillations have been linked to the tightly time-locked STN firings in response to NMDAergic cortico-subthalamic transmission (12). The fast kinetics of GluN2A NMDARs and their prominent somatic expres- sion could provide better temporal precision and larger positive currents near the axon hillock and thus facilitate the time-locked STN responses (12). This kinetic profile may also partly explain why the oscillations fall into beta frequencies, which are the frequency range of GluN2A kinetics $(12,21)$. Bursting cells such as STN neurons and Purkinje cells (PCs) were shown to have their CaT currents initiated in dendrites $(30,31)$. In theory, dendrites have less capacitance than soma and permit wider voltage fluctuations locally to unleash inactivated CaTs. Therefore, activation of GluN2B/D NMDARs in dendrites may initiate CaT-dependent bursts in STN. Although the causal relationship remains to be established, our data suggest that receptor distributions may contribute to the dissociation between bursts and oscillations.

Potential interactions between oscillation-based motor preparation and firing pattern-based motor execution in PD. Bradykinesia is characterized by slow movements beyond volitional control and therefore serves as a prototypical disease model of motor execution. Other than bradykinesia and STN bursts, the volitional motor controls, including motor decision or preparation, heavily modulate beta oscillations in PD $(4,7,32)$. This concept is supported by the observations in our free-moving paradigm, which revealed the typical shift of oscillatory frequencies (Figure $2 \mathrm{~J}$ and Figure $4 \mathrm{H}$ ) and the reduction of beta powers (Figure 2, $\mathrm{G}$ and $\mathrm{H}$, and Figure $4 \mathrm{G}$ ) when the rats decided to move, regardless of whether their motor performances changed due to CaT/NMDAR manipulations. STN has two parallel mechanisms, oscillations and firing patterns, to modulate movements. The interplay of the two mechanisms could explain how oscillation-based motor preparation tunes the firing pattern-based motor execution. Cortical oscillatory activities can be transmitted to STN via a GluN2A-mediated mechanism, which may oscillate the somatic membrane potentials in STN and disturb the precise timing of firing-pattern switches for motor execution. Motor preparation evidently desynchronizes cortical oscillatory activities $(4,7,33,34)$, which may suppress the above-mentioned processes and result in better motor execution. Similar mechanisms are well documented in the thalamus, the homolog of STN in developmental biology (35). Sleep induces thalamic oscillations and interferes with sensory information relays (36-38). Based on the fact that STN receives first-order command directly from the cortex via cortico-subthalamic pathway, it may be one of the fastest and the key mechanisms to explain how volitional motor preparation talks to the automatic/involuntary motor execution, and deserves further investigation by preparation-triggered protocols other than the free-moving paradigms in this study. By revisiting the oscillatory theory with the results in this study, parkinsonian motor control may be divided into two steps: the feedback, interactive oscillations for volitional motor decisions and preparations; and fast-responsive, feed-forward neuronal codes for involuntary motor execution, which results in bradykinesia. We did not investigate the interactions between beta oscillations and motor decisions in this study. However, PD patients have significant problems in decision making $(39,40)$, and this study provides the mechanism of beta oscillation that may help in further investigation of this issue.

The roles of neuronal codes versus oscillations in regulating normal motor behaviors. This study focused on the online circuitry mechanism of bradykinesia in PD. Notably, STN bursts and beta oscillations, which are pathologically augmented in PD, also exist 
in normal motor circuitry. Physiological amounts of STN bursts and beta oscillations are both present in normal conditions (12, 13). Reduction of cortical oscillatory activities in beta frequencies (beta desynchronization) is also observed in normal motor preparations $(33,34,41-43)$. Our study showed that the feed-forward, burst-coded mechanism also regulated inhibitory motor execution in rodents with intact basal ganglia circuits (Figures 3, 5, and 6, and Supplemental Figure 6) and is independent of oscillatory profiles. Naive rats also had cortical beta desynchronization in moving scenarios (Figure $3 \mathrm{H}$ ), regardless of whether the motor performances were being modulated by HC. The patterns and distributions of NMDARs and CaTs of STN in naive rodents were also similar to those in 6-OHDA rat models (Supplemental Figures 10 and 11). Beyond PD pathophysiology, these results may also apply to physiological states and improve our understandings of the fundamental principles of motor control physiology. Feedback circuitry oscillations may contribute to the volitional aspects of motor commands, while the feed-forward, firing pattern-coded neurotransmissions regulate motor execution (schematic summary, Supplemental Figure 12.

Therapeutic potential based on the new mechanism of bradykinesia. Our results shows that bradykinesia requires the collaboration between GluN2B/D NMDARs and CaTs in STN. Amantadine (44) and zonisamide (45), a weak NMDAR and a CaT blocker, respectively, already show modest clinical benefits in PD patients. However, potent and nonspecific NMDAR or CaT blockers are not ideal therapeutic options due to their cognitive side effects. In contrast, GluN2D (46) and $\mathrm{Ca}_{\mathrm{v}} 3.3$ (29) have low expression levels in the neocortex but are enriched in PD STN (Supplemental Figure 8 and 9). Regardless of the changes in dopaminergic system and direct-indirect pathways $(20,47)$, targeting of the neuron-modulatory consequences via GluN2D and $\mathrm{Ca}_{\mathrm{v}} 3.3$ may provide better therapeutic options. The standard dopaminergic therapy in PD is notorious for its motor complications (48). In contrast, NMDAR and $\mathrm{CaT}$ blockers did not induce the paradoxical rotations and head tilts (Figures 2 and 4) typically provoked by dopaminergic agents (12). Amantadine is the best-known anti-dyskinetic therapy in PD (44). In fact, intervention in the cortico-subthalamic pathway is the key mechanism of DBS $(12,13)$, and the therapeutic effect of DBS is better than the traditional therapy in terms of motor complications (49). Moreover, systemic dopaminergic therapy contributes to major cognitive and impulse control problems in PD $(40,48)$. Therefore, GluN2D and $\mathrm{Ca}_{\mathrm{v}} 3.3$ could be new bradykinesia-specific targets for PD motor therapy, and may be superior to the standard dopaminergic therapy in terms of its motor and cognitive complications.

\section{Methods}

Animals. Male adult Wistar rats were entered into the study at $\sim 8$ weeks of age and 250-350 g. 6-OHDA-lesioned (Sigma-Aldrich) hemiparkinsonian rats were used in all the PD experiments in this study (Supplemental Figure 1; see also Supplemental Methods). For optogenetics experiments, we used male adult Thy1-ChR2EYFP line 18 transgenic mice (catalog 007612; The Jackson Laboratory), which express channelrhodopsin-2 in cortical neuron layer V (13) and have been validated as an ideal animal model for selective stimulation of cortico-subthalamic axons $(12,13)$. Mice were entered into the study at $\sim 5$ weeks of age and weights greater than $20 \mathrm{~g}$. The animals were housed in a vivarium with controlled 12-hour dark/light cycles.

NMDAR and CaT modulators. We used NMDAR blockers with different subunit specificities. (D)-AP5 (2 mM, Tocris) is a non-selective NMDAR blocker. (R)-CPP (200 $\mu \mathrm{M}$, Tocris) is a selective NMDAR antagonist targeting the GluN2A subunit. Ro 25-6891 (RO; $1 \mathrm{mM}$, Tocris) and PPDA ( $500 \mu \mathrm{M}$, Tocris) inhibit GluN2B/D subunit selectively. To inhibit CaTs, we selected $\mathrm{NiCl}_{2}$ (6 mM, Sigma-Aldrich) and mibefradil ( $500 \mu \mathrm{M}$, Tocris). PPDA was dissolved in DMSO to $50 \mathrm{mM}$ first and then diluted with saline to achieve a final concentration of 500 $\mu \mathrm{M}$. All the other drugs were dissolved in artificial CSF (aCSF). The $\mathrm{pH}$ of all solutions was adjusted to 7.4.

Behavioral recordings and in vivo electrophysiology. We used the open-field test to evaluate the free-moving locomotor behaviors in 6-OHDA and control rodents, and the rotarod test for forced-moving behaviors (see Supplemental Methods for detailed paradigms). In valid 6-OHDA or normal control rats, we implanted microwire deep electrodes for single-unit and local field potential (LFP) recordings, as well as applying HCs. Epidural screw electrodes were also implanted for cortical LFPs. An STN cannula was inserted ipsilateral to 6-OHDA lesioning for real-time NMDAR or CaT modulations (see Supplemental Methods for all surgical procedures). We performed simultaneous behavioral and electrophysiological recordings, before and after online electric and/or pharmacological manipulations. Single-unit firings and LFPs were prefiltered and analyzed separately. For details, see Supplemental Methods.

Optic stimulation and simultaneous behavioral recordings. We activated the cortico-subthalamic axons optogenetically by implanting an optic fiber unilaterally into STN in Thy1-ChR2 mice (Figure 5A). We applied two different protocols: frequency-dependent $(10 \mathrm{~Hz})$ and frequency-independent (randomized) illumination (Figure 5B). Free-moving behaviors were accessed under baseline, light-off, and light-on states, with one of the stimulation protocols applied first by random process. For details, see Supplemental Methods.

Analysis of single-unit recordings and LFPs. Signals recorded for single-unit settings were post-processed with spike-sorting software (SciWorks 8.0, DataWave Technologies) and quality-controlled algorithm (12). Burst patterns were detected in each qualified single unit as described previously (10-12). The LFP data were post-processed with MATLAB 7.4 (MathWorks). Regional power spectrum represented in situ synchronizations, while coherence analysis referred to longranged synchronization. For details, see Supplemental Methods.

Immunohistochemistry. Three-month-old adult C57BL/6J mice and 4-month-old Wistar rats with or without 6-OHDA lesioning were used for immunohistochemistry study. Mice and rats were anesthetized with isoflurane and then sacrificed by an overdose of urethane ( $2 \mathrm{~g} / \mathrm{kg}$ i.p., Sigma-Aldrich) and transcardially perfused with $4 \%$ paraformaldehyde in PBS. The brain was then removed and immersed in the $4 \%$ paraformaldehyde overnight and moved to PBS for 3 days. The brain was sliced coronally at the thickness of $30 \mu \mathrm{m}$ by vibrotome. The sections were washed with PBS, followed by the suppression in $10 \%$ normal donkey serum in $0.1 \%$ Triton. The sections were subsequently incubated with respective primary antibodies overnight at $4{ }^{\circ} \mathrm{C}$ and then secondary fluorescent antibodies (all from Invitrogen). Primary antibodies included GluN2A (Neuromab, Cat. No. 75-288), GluN2B (Neuromab, 75-097), GluN2D (Bioss, Bs-1072R), MAP2 (Abcam, 
Ab5392), CaV3.1 (Alomone Labs, ACC-021), CaV3.2 (Alomone Labs, ACC-025) and CaV3.3 (Alomone Labs, ACC-009). Images were taken using confocal laser scanning microscope (Leica TCS SP2 two-photon microscope). See also Supplemental Methods and Supplemental Figure 13 for more details.

Statistics. The statistics were managed with SPSS 13.0 and plotted with Excel 2013 (Microsoft). Nonparametric Wilcoxon signed-rank test was used to analyze paired data, including animal behaviors, single-unit recordings, and LFP analyses (Figures 1-4 and Supplemental Figures 2-4). For those data with 3 or more conditions, we applied 1-way ANOVA with post-hoc Bonferroni correction (Figures 5 and 6 and Supplemental Figures $5-7$ ). In all statistical methods, a $P$ value less than 0.05 was considered significant.

Study approval. The study was approved by the IACUC of National Taiwan University College of Medicine and College of Public Health.

\section{Author contributions}

MKP designed the study and generated the behavioral and electrophysiological results in rats with CHT, YMW, WCL, and TRW. SHK designed and generated the histology/pathology data in rodents and human subjects. MKP and WSL designed the optogenetic experiments in mice and analyzed the results with JCP and CYC. MKP and JYL designed MATLAB codes. CCK led the team and coordinated the study. MKP and CCK interpreted the results and wrote the article with input and comments from the other authors.

\section{Acknowledgments}

We thank F.-C. Lin (Southport Tech. Co.) for the technical support. This research is supported by the Ministry of Science and Technology in Taiwan (MOST-103-2320-B-002-026-MY3 and MOST104-2321-B-002-067 to CCK; MOST-104-2314-B-002-076-MY3 to MKP), the National Health Research Institute in Taiwan (NHRIEX105-10503NI to CCK); National Taiwan University Hospital (104-N2870 and MG380 to MKP) and the Yin-Lin branch of the hospital (NTUHYL104.N007 to MKP and YMW).

Address correspondence to: Chung-Chin Kuo, Departments of Physiology and Neurology, National Taiwan University College of Medicine, No. 1, Sec. 1, Ren-Ai Road, Taipei 100, Taiwan. Phone: 886.2.23123456 ext.88236; E-mail: chungchinkuo@ntu.edu.tw.
1. Sharott A, Magill PJ, Harnack D, Kupsch A, Meissner W, Brown P. Dopamine depletion increases the power and coherence of beta-oscillations in the cerebral cortex and subthalamic nucleus of the awake rat. Eur J Neurosci. 2005;21(5):1413-1422.

2. Moran RJ, et al. Alterations in brain connectivity underlying beta oscillations in Parkinsonism. PLoS Comput Biol. 2011;7(8):e1002124.

3. Cassidy M, et al. Movement-related changes in synchronization in the human basal ganglia. Brain. 2002;125(Pt 6):1235-1246.

4. Kühn AA, et al. Event-related beta desynchronization in human subthalamic nucleus correlates with motor performance. Brain. 2004;127(Pt 4):735-746.

5. de Hemptinne C, et al. Therapeutic deep brain stimulation reduces cortical phase-amplitude coupling in Parkinson's disease. Nat Neurosci. 2015;18(5):779-786.

6. Pogosyan A, et al. Parkinsonian impairment correlates with spatially extensive subthalamic oscillatory synchronization. Neuroscience. 2010;171(1):245-257.

7. Williams D, et al. The relationship between oscillatory activity and motor reaction time in the parkinsonian subthalamic nucleus. Eur J Neurosci. 2005;21(1):249-258.

8. Chen CC, et al. Complexity of subthalamic 13-35 $\mathrm{Hz}$ oscillatory activity directly correlates with clinical impairment in patients with Parkinson's disease. Exp Neurol. 2010;224(1):234-240.

9. Loukas C, Brown P. Online prediction of selfpaced hand-movements from subthalamic activity using neural networks in Parkinson's disease. J Neurosci Methods. 2004;137(2):193-205.

10. Tai CH, Pan MK, Lin JJ, Huang CS, Yang YC, Kuo CC. Subthalamic discharges as a causal determinant of parkinsonian motor deficits. Ann Neurol. 2012;72(3):464-476.

11. Tai CH, Yang YC, Pan MK, Huang CS, Kuo CC. Modulation of subthalamic T-type $\mathrm{Ca}(2+)$ channels remedies locomotor deficits in a rat model of Parkinson disease. J Clin Invest.
2011;121(8):3289-3305.

12. Pan MK, Tai CH, Liu WC, Pei JC, Lai WS, Kuo CC. Deranged NMDAergic cortico-subthalamic transmission underlies parkinsonian motor deficits. J Clin Invest. 2014;124(10):4629-4641.

13. Gradinaru V, Mogri M, Thompson KR, Henderson JM, Deisseroth K. Optical deconstruction of parkinsonian neural circuitry. Science. 2009;324(5925):354-359.

14. Pogosyan A, Gaynor LD, Eusebio A, Brown P. Boosting cortical activity at Beta-band frequencies slows movement in humans. Curr Biol. 2009;19(19):1637-1641.

15. Krause V, Wach C, Südmeyer M, Ferrea S, Schnitzler A, Pollok B. Cortico-muscular coupling and motor performance are modulated by $20 \mathrm{~Hz}$ transcranial alternating current stimulation (tACS) in Parkinson's disease. Front Hum Neurosci. 2013;7:928.

16. Chen CC, et al. Excessive synchronization of basal ganglia neurons at $20 \mathrm{~Hz}$ slows movement in Parkinson's disease. Exp Neurol. 2007;205(1):214-221.

17. Fogelson N, et al. Frequency dependent effects of subthalamic nucleus stimulation in Parkinson's disease. Neurosci Lett. 2005;382(1-2):5-9.

18. Little $\mathrm{S}$, et al. Adaptive deep brain stimulation in advanced Parkinson disease. Ann Neurol. 2013;74(3):449-457.

19. Beurrier C, Congar P, Bioulac B, Hammond C. Subthalamic nucleus neurons switch from single-spike activity to burst-firing mode. J Neurosci. 1999;19(2):599-609.

20. Kravitz AV, et al. Regulation of parkinsonian motor behaviours by optogenetic control of basal ganglia circuitry. Nature. 2010;466(7306):622-626.

21. Perouansky M, Yaari Y. Kinetic properties of NMDA receptor-mediated synaptic currents in rat hippocampal pyramidal cells versus interneurones. J Physiol (Lond). 1993;465:223-244.

22. Schmidt R, Leventhal DK, Mallet N, Chen F, Berke JD. Canceling actions involves a race between basal ganglia pathways. Nat Neurosci. 2013;16(8):1118-1124.

23. Sanders TH, Jaeger D. Optogenetic stimulation of cortico-subthalamic projections is sufficient to ameliorate bradykinesia in 6-ohda lesioned mice. Neurobiol Dis. 2016;95:225-237.

24. Li Q, et al. Therapeutic deep brain stimulation in Parkinsonian rats directly influences motor cortex. Neuron. 2012;76(5):1030-1041.

25. Parker PR, Lalive AL, Kreitzer AC. Pathway-Specific Remodeling of Thalamostriatal Synapses in Parkinsonian Mice. Neuron. 2016;89(4):734-740.

26. Fuentes R, Petersson P, Siesser WB, Caron MG, Nicolelis MA. Spinal cord stimulation restores locomotion in animal models of Parkinson's disease. Science. 2009;323(5921):1578-1582.

27. Delrobaei M, Tran S, Gilmore G, McIsaac K, Jog M. Characterization of multi-joint upper limb movements in a single task to assess bradykinesia. J Neurol Sci. 2016;368:337-342.

28. Perez FA, Palmiter RD. Parkin-deficient mice are not a robust model of parkinsonism. Proc Natl Acad Sci U S A. 2005;102(6):2174-2179.

29. Talley EM, Cribbs LL, Lee JH, Daud A, PerezReyes E, Bayliss DA. Differential distribution of three members of a gene family encoding low voltage-activated (T-type) calcium channels. JNeurosci. 1999;19(6):1895-1911.

30. Song WJ, Baba Y, Otsuka T, Murakami F. Characterization of $\mathrm{Ca}(2+)$ channels in rat subthalamic nucleus neurons. J Neurophysiol. 2000;84(5):2630-2637.

31. Otsu Y, et al. Activity-dependent gating of calcium spikes by A-type $\mathrm{K}+$ channels controls climbing fiber signaling in Purkinje cell dendrites. Neuron. 2014;84(1):137-151.

32. Williams D, et al. Behavioural cues are associated with modulations of synchronous oscillations in the human subthalamic nucleus. Brain. 2003;126(Pt 9):1975-1985.

33. Meyniel F, Pessiglione M. Better get back to work: a role for motor beta desynchronization in incen- 
tive motivation. J Neurosci. 2014;34(1):1-9.

34. Rubino D, Robbins KA, Hatsopoulos NG. Propagating waves mediate information transfer in the motor cortex. Nat Neurosci. 2006;9(12):1549-1557.

35. Marani E, Heida T, Lakke EA, Usunoff KG. The subthalamic nucleus. Part I: development, cytology, topography and connections. Adv Anat Embryol Cell Biol. 2008;198:1-113, vii.

36. Schabus $\mathrm{M}$, et al. The fate of uncoming stimuli during NREM sleep is determined by spindles and the phase of the slow oscillation. Front Neurol. 2012;3:40.

37. Palva S, Linkenkaer-Hansen K, Näätänen R, Palva JM. Early neural correlates of conscious somatosensory perception. J Neurosci. 2005;25(21):5248-5258.

38. Boly M, et al. Baseline brain activity fluctuations predict somatosensory perception in humans. Proc Natl Acad Sci U S A. 2007;104(29):12187-12192.

39. Voon V, et al. Mechanisms underlying dopa- mine-mediated reward bias in compulsive behaviors. Neuron. 2010;65(1):135-142.

40. Callesen MB, Scheel-Krüger J, Kringelbach ML, Møller A. A systematic review of impulse control disorders in Parkinson's disease. J Parkinsons Dis. 2013;3(2):105-138.

41. Khanna P, Carmena JM. Neural oscillations: beta band activity across motor networks. Curr Opin Neurobiol. 2015;32:60-67.

42. Babiloni C, et al. Alpha, beta and gamma electrocorticographic rhythms in somatosensory, motor, premotor and prefrontal cortical areas differ in movement execution and observation in humans. Clin Neurophysiol. 2016;127(1):641-654.

43. Sanes JN, Donoghue JP. Oscillations in local field potentials of the primate motor cortex during voluntary movement. Proc Natl Acad Sci U S A. 1993;90(10):4470-4474.

44. Hubsher G, Haider M, Okun MS. Amantadine: the journey from fighting flu to treating Parkin- son disease. Neurology. 2012;78(14):1096-1099.

45. Yang YC, Tai CH, Pan MK, Kuo CC. The T-type calcium channel as a new therapeutic target for Parkinson's disease. Pflugers Arch. 2014;466(4):747-755.

46. Laurie DJ, Bartke I, Schoepfer R, Naujoks K, Seeburg PH. Regional, developmental and interspecies expression of the four NMDAR2 subunits, examined using monoclonal antibodies. Brain Res Mol Brain Res. 1997;51(1-2):23-32.

47. Zhuang X, Mazzoni P, Kang UJ. The role of neuroplasticity in dopaminergic therapy for Parkinson disease. Nat Rev Neurol. 2013;9(5):248-256.

48. Bastide MF, et al. Pathophysiology of L-dopainduced motor and non-motor complications in Parkinson's disease. Prog Neurobiol. 2015; 132:96-168.

49. Schuepbach WM, et al. Neurostimulation for Parkinson's disease with early motor complications. NEngl JMed. 2013;368(7):610-622. 